\title{
Rapid Regression of Obstructive Cardiac Rhabdomyoma in a Preterm Neonate after Sirolimus Therapy
}

\author{
Seung Jae Lee ${ }^{a}$ Eun Song Song ${ }^{a}$ Hwa Jin Cho ${ }^{a}$ Young Youn Choi ${ }^{a}$ \\ Jae Sook Mab Young Kuk Cho ${ }^{a}$ \\ a Department of Pediatrics, Chonnam National University Hospital, Chonnam National University \\ Medical School, and ${ }^{\text {b}}$ Department of Pediatrics, KS Hospital, Gwangju, South Korea
}

\section{What Is It about?}

Most cardiac rhabdomyomas become smaller with time; however, emergency intervention is indicated when severe obstruction induces hemodynamic instability. Mammalian target of rapamycin (mTOR) inhibitors have been used to treat neonates and children with hemodynamically obstructive cardiac rhabdomyoma. Herein, we report a premature neonate with severe left ventricular outflow tract obstructive cardiac rhabdomyoma who was successfully treated with the mTOR inhibitor sirolimus. Sirolimus could be considered as an alternative medical option for managing premature neonates with obstructive cardiac rhabdomyoma.

\section{Established Facts}

- Mammalian target of rapamycin (mTOR) inhibitors have been used to treat neonates and children with hemodynamically obstructive cardiac rhabdomyoma.

\section{Novel Insights}

- This is the first recorded case of a premature neonate with obstructive cardiac rhabdomyoma who has been successfully treated with an mTOR inhibitor.

- Sirolimus (mTOR inhibitor) could be considered as an alternative medical option for managing premature neonates with obstructive cardiac rhabdomyoma. 


\title{
Keywords
}

Cardiac rhabdomyoma $\cdot$ Left ventricle inlet obstruction $\cdot$ Tuberous sclerosis

\begin{abstract}
Cardiac rhabdomyoma can be subclinical or fatal depending on the onset age, involving site, and the size and degree of invasion. Although most rhabdomyomas become smaller with time, emergency intervention is indicated when severe obstruction induces hemodynamic instability. Mammalian target of rapamycin (mTOR) inhibitors have been used to treat neonates and children with hemodynamically obstructive cardiac rhabdomyoma. Herein, we report a premature neonate at the gestational age of $30+4$ weeks with severe left ventricular outflow tract obstructive cardiac rhabdomyoma who was successfully treated with the mTOR inhibitor sirolimus. To the best of our knowledge, this is the first recorded case of a premature neonate with obstructive cardiac rhabdomyoma who was successfully treated with an mTOR inhibitor. Therefore, sirolimus could be considered as an alternative medical option for managing premature neonates with obstructive cardiac rhabdomyoma.
\end{abstract}

(C) 2017 The Author(s)

Published by S. Karger AG, Basel

\section{Introduction}

Tuberous sclerosis is a well-recognized multisystem genetic disease that causes benign tumors to grow in various organs such as kidney, heart, eye, lung, and skin [1]. Among them, multiple intracardiac rhabdomyomas can be easily discovered in a newborn with tuberous sclerosis [2]. Intracardiac rhabdomyoma can be subclinical or fatal depending on the onset age, the involving site, and the size and degree of invasion [3-5]. Although most rhabdomyomas in early life become smaller with time, emergency intervention is indicated when severe obstruction induces hemodynamic instability [6, 7].

Mammalian target of rapamycin (mTOR) inhibitors have been used to treat neonates and children with hemodynamically obstructive cardiac rhabdomyoma [8-10]. Herein, we report a premature neonate at the gestational age (GA) of $30+4$ weeks with severe left ventricular outflow tract obstructive cardiac rhabdomyoma who was successfully treated with the MTOR inhibitor sirolimus.

\section{Case Report}

A preterm male infant was born at the GA of 28 weeks by vaginal delivery due to prolonged premature rupture of membrane. The patient was the second baby of healthy nonconsanguineous parents, a 36-year-old male and a 33-year-old female. His mother and brother had been diagnosed with tuberous sclerosis. His mother was transferred to our hospital due to premature rupture of membrane. Fetal multiple cardiac rhabdomyomas were detected in fetal echocardiography at the GA of 25 weeks. Soon after delivery at the GA of 28 weeks, he had weak crying, lip cyanosis, and low muscle tone. He was admitted for premature care and evaluation of cardiac tumors. The American Pediatric Gross Assessment Record (APGAR) score was 5 at $1 \mathrm{~min}$ and 6 at $5 \mathrm{~min}$. He weighed 1,170 g (50-75 percentile), with a body length of $39.0 \mathrm{~cm}$ (50-75 percentile), head circumference of $27.0 \mathrm{~cm}$ (50-75 percentile), respiratory rate of 70-75 breaths/min, heart rate of 140 beats/min, blood pressure of $45 / 38 \mathrm{~mm} \mathrm{Hg}$, and oxygen saturation of $75-80 \%$. Chest X-ray revealed acute respiratory distress syndrome and right tension pneumothorax. Therefore, he was given mechanical ventilation support. He also received surfactant therapy and right chest tubing. On the third day after birth, the chest tube 

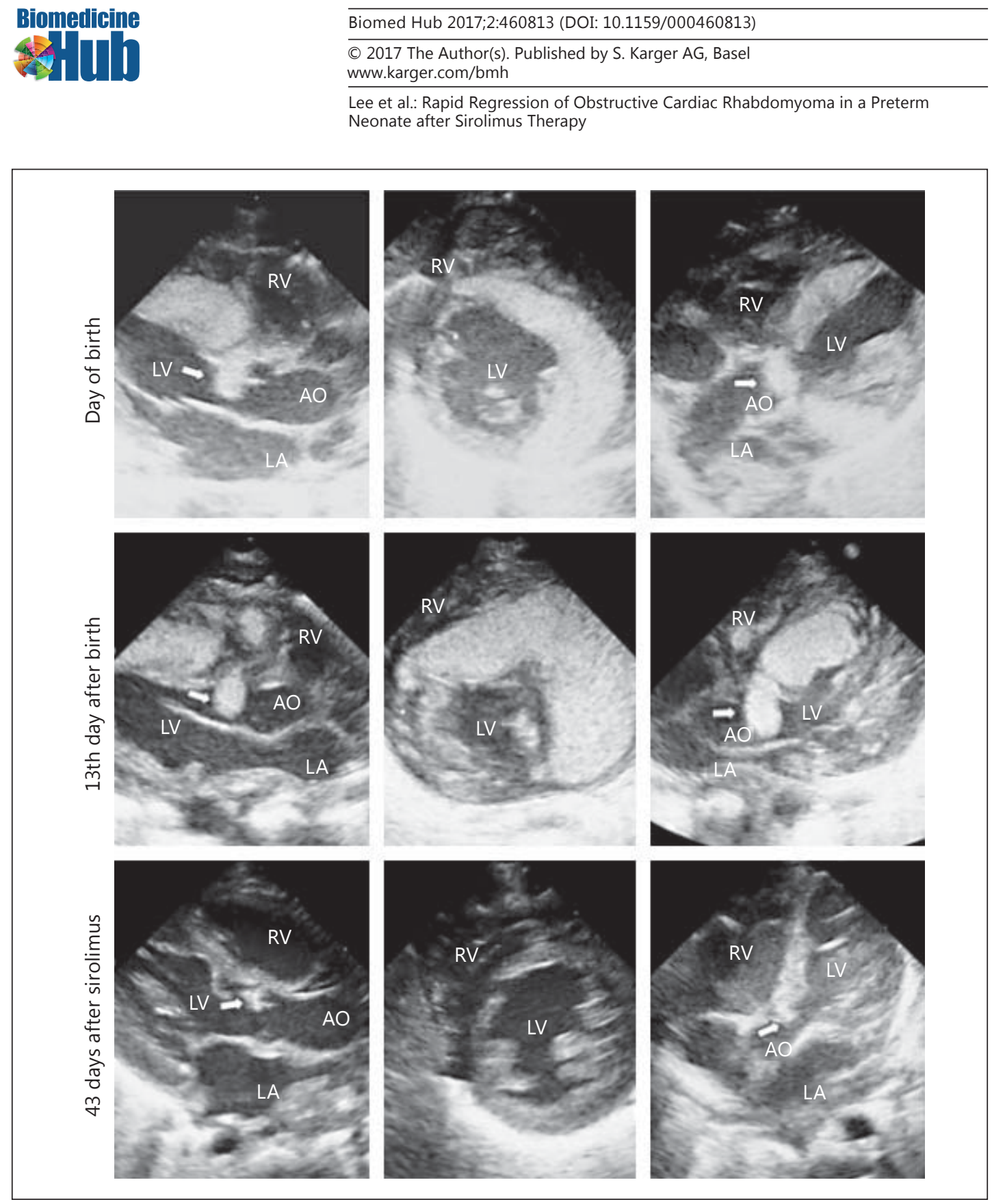

Fig. 1. Echocardiographic images of cardiac rhabdomyomas from parasternal long-axis (left column), parasternal short-axis (middle column), and apical 5-chamber (right column) views. The top row of images was taken on the day of birth. The middle row of images was taken on the 13th day after birth. The bottom row of images was taken at 43 days after initiating sirolimus. LA, left atrium; LV, left ventricle; RV, right ventricle; AO, aorta; arrows, subaortic mass.

was removed. Physical examination revealed no definitive skin features suggesting tuberous sclerosis such as facial angiofibromas or hypomelanotic macules. Cranial ultrasound showed a grade 1 intraventricular hemorrhage without tuber. A grade 2/6 systolic murmur was found at the left upper sternal border.

On the day of birth, echocardiogram revealed rhabdomyomas of various sizes with multiple locations, including a huge 13.2-mm rhabdomyoma located in the left ventricle (LV) free wall to interventricular septum, a $4.9 \times 3.3-\mathrm{mm}$ subaortic interventricular septum, and a 5.1-mm rhabdomyoma in the LV free wall and the right ventricle (RV) apex area (Fig. 1). 
Fig. 2. During sirolimus treatment, serum levels of sirolimus and white blood cell (WBC) counts were monitored at neonatal intensive care unit admission.

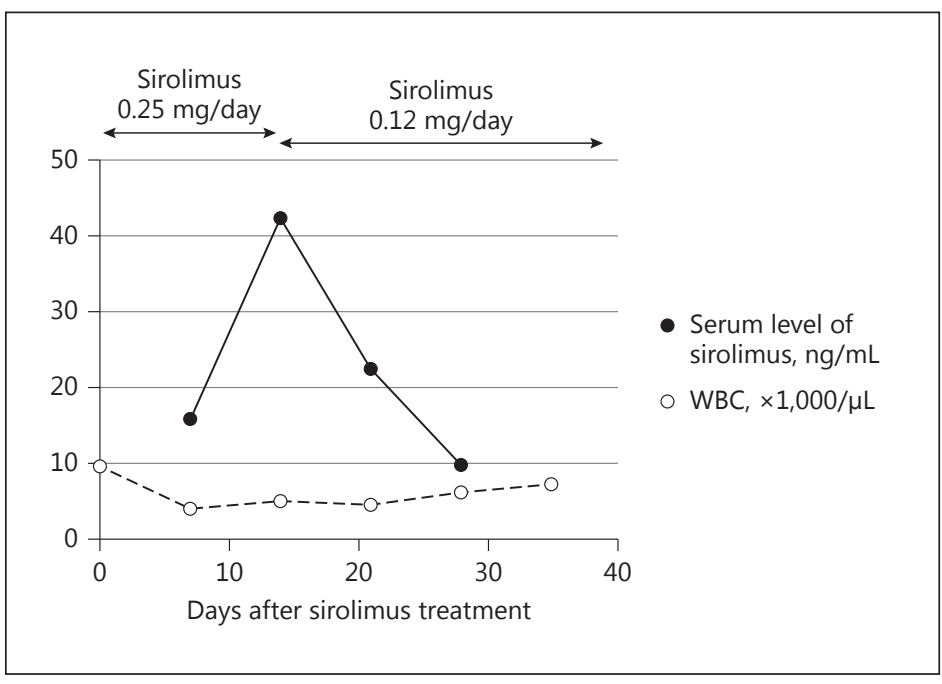

There was severe LV outflow tract obstruction (about 80\%) due to subaortic rhabdomyoma. There was also very small patent ductus arteriosus. Blood pressure was stable after discontinuation of low-dose dobutamine infusion which lasted 1 day.

At 4 days after birth, he suddenly had chest retraction, tachypnea, and severe bradycardia. Chest X-ray showed pneumomediastinum, subcutaneous emphysema, and right tension pneumothorax. The right chest tube was reinserted to improve the pneumomediastinum and right tension pneumothorax. Saline was loaded and dopamine was added to maintain blood pressure and support cardiac function. After cardiopulmonary resuscitation for $40 \mathrm{~min}$, he returned to spontaneous circulation. After 5 days of cardiopulmonary resuscitation, the chest tube was removed and his vital signs were stable.

At 13 days after birth, echocardiography showed increased-sized subaortic rhabdomyoma $(5.2 \times 3.6 \mathrm{~mm})$, which caused the aggravation of LV outflow obstruction. RV inlet obstruction also developed due to enlarged interventricular septal rhabdomyoma and a newly developed RV free wall mass. The LV function by M-mode echocardiography was decreased due to dyskinetic hypertrophied interventricular septum to the LV free wall (Fig. 1).

These large and multiple cardiac rhabdomyomas were ineligible for surgical resection because of preterm low body weight of $1,200 \mathrm{~g}$ with extensive intramural myocardial involvement.

Therefore, oral sirolimus $0.25 \mathrm{mg}$, an mTOR inhibitor, was given to him once daily starting on the 18th day after birth (GA $30+4$ weeks). We adjusted the dose according to the study by Breathnach et al. [10]. We monitored his complete blood cell count and electrolytes. Hepatic and renal function tests as well as lipid profiling were also performed. The results are shown in Figure 2. Prophylaxis with co-trimoxazole was used during sirolimus treatment. There was no event of infection. We also monitored serum levels of sirolimus and achieved a steady-state serum level of the drug at $10-20 \mathrm{ng} / \mathrm{mL}$.

At 14 days after mTOR treatment, we tapered sirolimus to $0.12 \mathrm{mg}$ once daily due to high serum levels of sirolimus $(42.1 \mathrm{ng} / \mathrm{ml})$. Serial echocardiography was performed to evaluate the tumor size. At 15 days after mTOR treatment, the subaortic obstructive tumor started to decrease in size $(4.7 \times 3.1 \mathrm{~mm})$. On the $22 \mathrm{nd}$ day of treatment, there was a dramatic reduction in size $(3.7 \times 3.0 \mathrm{~mm})$. On the $43 \mathrm{rd}$ day of treatment, the $\mathrm{LV}$ outflow tract tumor decreased to a size of $2.3 \times 1.9 \mathrm{~mm}$. The interventricular septal mass became smaller $(8.7 \mathrm{~mm})$ and nearly 
lost its echogenicity at 43 days after mTOR treatment (Fig. 1). Other rhabdomyomas were also improved in the LV superior area, RV apex area, and RV free wall area. The obstruction in the RV inflow also disappeared at 43 days after mTOR treatment.

At 2 months of age (43 days after the mTOR treatment), the patient was discharged with sirolimus for 2 weeks. His body weight was 2,230 g. At 2 months and 2 weeks of age, we stopped sirolimus treatment in the outpatient department to prevent side effects from the drug. He has been symptom-free for 7 months under follow-up in the outpatient department.

\section{Discussion}

Tuberous sclerosis complex (TSC) is caused by an inactivating mutation of the TSC1 (chromosome 9q34.3; protein hamartin) or TSC2 (chromosome 16p13.3; protein tuberin) gene. TSC1 and TSC2 genes can act as tumor growth suppressors. They control cell proliferation and differentiation, thus influencing mTOR function $[1,2]$. Mutations in these genes can lead to hyperactivation of the mTOR signal pathway and subsequent abnormalities in various organs $[1,2]$.

Clinical presentation of patients with rhabdomyomas of the heart in TSC can vary, ranging from asymptomatic to arrhythmias, valvular obstruction, and life-threatening congestive heart failure [8]. In our case, the patient had chest retraction, tachypnea, and bradycardia. Spontaneous circulation was successfully achieved after cardiopulmonary resuscitation. Inotropic agents were added to support cardiac function. The patient also had mechanical ventilation support. Although most rhabdomyomas in early life can become smaller with time, in our case, severe obstruction induced hemodynamic instability. The risk involved with surgical intervention seemed to be too high because of the preterm low body weight of $1,200 \mathrm{~g}$ with extensive multiple intramural myocardial involvement.

The mTOR inhibitor sirolimus has been used as an immunosuppressant in cardiac and kidney transplant recipients. Gradually, everolimus has been used to treat renal angiomyolipomas and subependymal giant cell astrocytomas in patients with tuberous sclerosis $[8,9]$. Recently, sirolimus and everolimus have been used as treatment in a full-term neonate and a child with cardiac rhabdomyoma under inoperable conditions [8-10].

Sirolimus and everolimus treatment can cause dyslipidemia and decrease hepatic and renal functions and immunosuppression. Therefore, these parameters should be carefully monitored. Prophylaxis with co-trimoxazole has been recommended $[9,10]$. We monitored the serum levels of sirolimus and achieved a steady-state serum level of the drug at 10-20 $\mathrm{ng} / \mathrm{mL}$. An initial dosage of oral sirolimus $0.25 \mathrm{mg}$ once daily was chosen to avoid toxicity while maintaining therapeutic levels. It was adjusted based on the dose of $0.5 \mathrm{mg}$ once daily for full-term neonates used in the study by Breathnach et al. [10]. However, sirolimus level was elevated in our patient to $42.1 \mathrm{ng} / \mathrm{mL}$. Therefore, we tapered sirolimus to $0.12 \mathrm{mg}$ once daily. Tumor regression was observed with this dosage. Complete blood cell count, electrolytes, hepatic and renal functions, and lipid profiling results were found to be normal at this dose. Based on our data from sirolimus level monitoring, $0.12 \mathrm{mg}$ of sirolimus once daily might be the optimal dosage for premature neonates with a body weight of 1,000-2,000 g.

To date, only a few reports have described the use of an mTOR inhibitor as treatment for neonates and children with cardiac rhabdomyoma [8-10]. To the best of our knowledge, this is the first report of a premature neonate with a GA of $30+4$ weeks and body weight of $1,200 \mathrm{~g}$ and severe obstructive cardiac rhabdomyoma who was successfully treated with the mTOR inhibitor sirolimus. There may be differences in metabolic disposition and pharmacokinetics of sirolimus between full-term and premature neonates. However, long-term pediatric risks for premature neonates from sirolimus-based immunosuppression are not well 
known, and the mechanistic basis of age-dependent changes in sirolimus has not yet been fully defined [11]. We monitored his complete blood cell count and electrolytes. Hepatic and renal function tests as well as lipid profiling were also performed. The results are shown in Figure 2. Prophylaxis with co-trimoxazole was used during sirolimus treatment. There was no event of infection. In this case, we found a successful treatment in a premature neonate weighing 1,200 $\mathrm{g}$ without side effects from sirolimus such as bacterial infection, dyslipidemia, myelosuppression, and renal dysfunction. We achieved a steady-state serum level of sirolimus at $10-20 \mathrm{ng} / \mathrm{mL}$ under $0.12 \mathrm{mg}$ once daily.

Although additional studies should be performed to confirm the safety and effectiveness of sirolimus in premature neonates, sirolimus might be considered as an alternative medical option for managing premature neonates with obstructive cardiac rhabdomyoma.

\section{Ethics Statement}

We obtained ethical review board approval for this study (CNUH-EXP-2017-018).

\section{Disclosure Statement}

This study was supported by a grant (NRF-2015R1D1A1A01059017) of the Basic Science Research Program through the National Research Foundation of Korea (NRF) funded by the Ministry of Education, Republic of Korea. The authors declare that they have no competing interests.

\section{References}

1 Borkowska J, Schwartz RA, Kotulska K, Jozwiak S: Tuberous sclerosis complex: tumors and tumorigenesis. Int J Dermatol 2011;50:13-20.

-2 Northrup H, Krueger DA; International Tuberous Sclerosis Complex Consensus Group: Tuberous sclerosis complex diagnostic criteria update: recommendations of the 2012 International Tuberous Sclerosis Complex Consensus Conference. Pediatr Neurol 2013;49:243-254.

-3 Arnaiz G, Toledo G, Borzutzky S, Urcelay M, Heusser R, Garay G, Castillo N, Toro R, Becker R, Arretz V: Cardiac tumors in children and adults: a multicentric retrospective study (in Spanish). Rev Med Chil 2006;134:11351145 .

4 Freedom R, Lee KJ, MacDonald C, Taylor G: Selected aspects of cardiac tumors in infancy and childhood. Pediatr Cardiol 2000;21:299-316.

5 Black MD, Kadletz M, Smallhorn JF, Freedom RM: Cardiac rhabdomyomas and obstructive left heart disease: histologically but not functionally benign. Ann Thorac Surg 1998;65:1388-1390.

6 Karnak İ, Alehan D, Ekinci S, Büyükpamukçu N: Cardiac rhabdomyoma as an unusual mediastinal mass in a newborn. Pediatr Surg Int 2007;23:811-814.

7 Padalino MA, Reffo E, Cerutti A, Favero V, Biffanti R, Vida V, Stellin G, Milanesi O: Medical and surgical management of primary cardiac tumours in infants and children. Cardiol Young 2014;24:268-274.

-8 Tiberio D, Franz DN, Phillips JR: Regression of a cardiac rhabdomyoma in a patient receiving everolimus. Pediatrics 2011;127:e1335-e1337.

9 Demir HA, Ekici F, Erdem AY, Emir S, Tunç B: Everolimus: a challenging drug in the treatment of multifocal inoperable cardiac rhabdomyoma. Pediatrics 2012;130:e243-e247.

10 Breathnach C, Pears J, Franklin O, Webb D, McMahon CJ: Rapid regression of left ventricular outflow tract rhabdomyoma after sirolimus therapy. Pediatrics 2014;134:e1199-e1202.

11 Emoto C, Fukuda T, Mizuno T, Schniedewind B, Christians U, Adams D, Vinks A: Characterizing the developmental trajectory of sirolimus clearance in neonates and infants. CPT Pharmacometrics Syst Pharmacol 2016; 5:411-417. 